Mitteilungen aus dem biologischen Süßwasserlaboratorium Frederiksdal bei Lyngby (Dänemark). Nr. V.

\title{
Über die Biologie von Glyphotaelius punctatolineatus Retz. nebst Bemerkungen über das freilebende Puppen- stadium der Wasserinsekten.
}

\author{
Von \\ Dr. C. Wesenberg-Lund (Hilleröd). \\ Mit. Tafel I (Figur 1-32) und einer Abbildung im Text.
}

\section{Naturbeobachtungen über Glyphotaelius.}

An den Ufern des Versuchsteichs II findet man in den letzten Tagen des Juni bis ca. 1. August auf der Ufervegetation große, fahle Gallertmassen (Fig. 1); sie sind von länglicher Form, beinahe 30 bis $40 \mathrm{~cm}$ lang; ca. $1 \mathrm{~cm}$ breit und ca. $3 / 4$ bis $1 \mathrm{~cm}$ hoch. Das sind die Eiermassen von Glyphotaelius punctatolineatus Retz., der nach E. Petersen einer unserer seltenen Phryganeen ist (1907, S. 145). Betrachtet man eben abgelegte Eiermassen, sieht man, daß sie quergefurcht sind; später, wenn die Gallerte älter werden, und besonders wenn die Jungen ausgekrochen sind, geht die Querfurchung verloren. Ich habe leider nie die Eiablage beobachtet, und so viel ich weib, hat auch kein anderer sie gesehen. Weil ich aber sehr oft in der Eiablageperiode an den Ufern der Teiche war, recht häufig das Tier sah, nie aber eine Eiablage, vermute ich, daß diese in der Abenddämmerung oder in der Nacht vor sich geht. Wahrscheinlich stammt die Querfurchung davon her, daß das Weibchen, wenn die Eier hervortreten, die Spitze des Abdomens von links nach rechts oder umgekehrt bewegt, indem es zugleich langsam an dem Stengel aufoder niederwärts wandert.

Im Jahre 1907 fanden die Eier sich ca. am 1. Juli, im Jahre 1909 ca. am 10. Juli in großen Mengen. Die Eiermassen waren immer über dem Wasser angebracht, öfters an den Irisblättern, aber auch auf Carexhalmen u. a. Sehr oft standen die Pflanzen direkt im Wasser, oft aber am Ufer wachsend und bis ca. $1 / 2 \mathrm{~m}$ vom Wasser entfernt. In Trocken- 
perioden werden die Eiermassen milchweiß, und die äußersten Gallertschichten werden dann dick und sehr zähflüssig; ferner beobachtet man, daß das Volumen stark abnimmt. In Regenperioden quellen sie stark auf, die Gallertmassen werden beinahe hyalin und sehr flüssig. In den Trockenperioden hört die Entwicklung der Eier ganz auf, dagegen entwickeln die Eier sich in den Regenperioden mit merkwürdiger Schnelligkeit. 28. Juni wurden die Blätter, worauf vier wahrscheinlich eben abgelegte Eiermassen saßen, markiert und alle drei Tage besichtigt. In den feuchten, recht

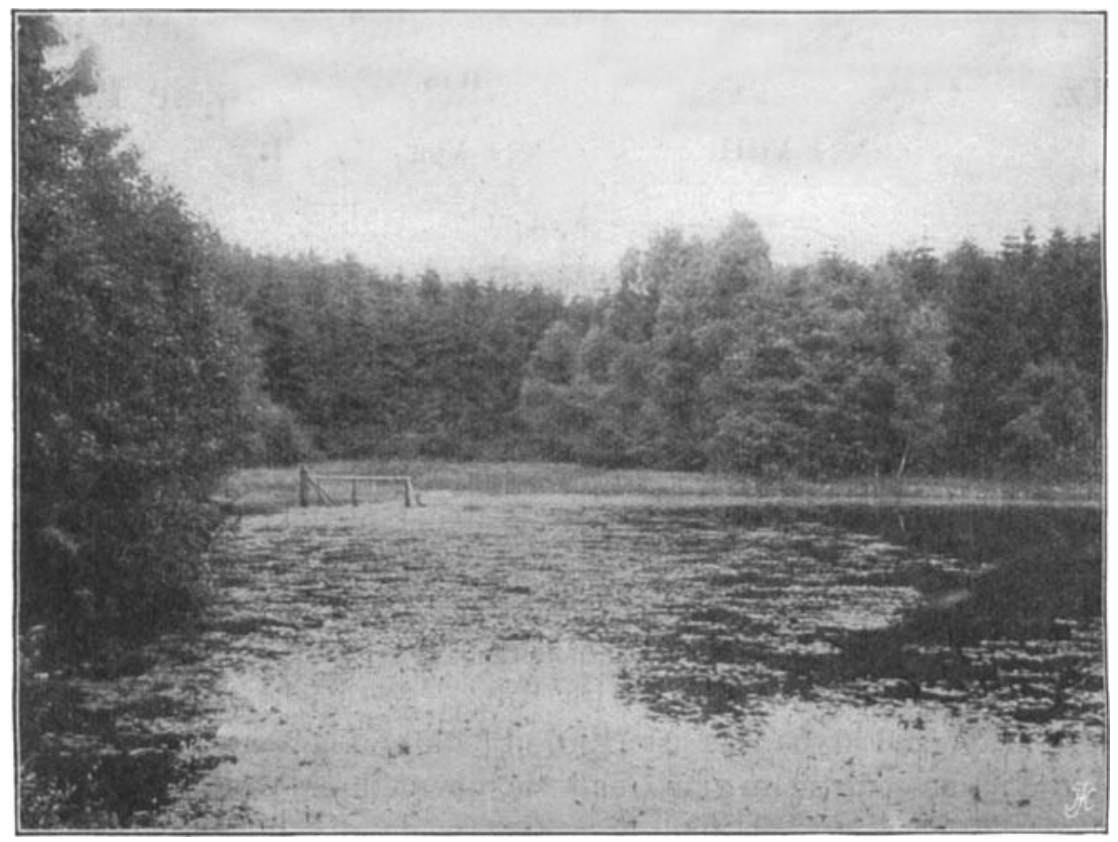

Versuchsteich II; die Heimat der Glyphotaelius, die Wasseroberfläche mit Potamogeton natans bedeckt. (Winge phot.)

kalten Tagen wuchsen sie beträchtlich. 10. Juli enthielten die Klumpen kleine Larven, die sich noch nicht bewegen konnten. 13. Juli saßen die Gallertmassen noch auf den Blättern, waren aber von Larven ganz entleert.

Ich habe Gallertmassen genommen und an eine trockene, von der Sonne beschienene Stelle gebracht; 14 Tage verhielten die Eiermassen sich ganz unverändert. Dann wurden die nun verwelkten Blätter mit den Eiermassen in Wasser unter eine Glasglocke gestellt; die Gallerten quollen auf, die früher weißgelben Eier wurden schwarz, und im Laufe von fünf Tagen krochen die Larven aus. 20. bis 25. Juli fand ich überall zerfließende Gallertmassen. Die Larven halten sich, besonders in Trocken- 
perioden, in den Gallerten auf, wachsen und nähren sich unzweifelhaft von denselben. Bald findet man in den Gallertmassen Eierschalen, bald nicht; im letzteren Falle sind sie von den Larven verzehrt.

In den herabfließenden Laichmassen sammeln die Larven sich immer in den niedrigsten 'Teilen und fließen mit denselben langsam hinunter. Eiermassen, die so aufgehängt waren, daß die Larven direkt ins Wasser niedertropfen konnten, habe ich nie gesehen, glaube aber, daß der Transport der Larven besonders in den Morgenstunden, wenn der Tau alles bedeckt, wie auch im Regen vor sich geht.

Wenn die Eiermassen an vertikalen, direkt im Wasser wachsenden Binsenstengeln abgesetzt wurden, flossen die Larven natürlich mit den Gallerten direkt in das Wasser hinab. Häufig aber konnten die Larven während des passiven Transports nicht das Wasser, sondern nur die feste Erde, und das oft in einer Entfernung von cirea $1 \mathrm{~m}$ vom Wasser, orreichen.

So weit ich verstehen kann, sind auch die Larven (Fig. 26-27) für eine direkte Wanderung angepaßt; darauf deuten besonders die relativ sehr langen Beine, von welchen die letzten zwei Paare beinahe eine halbe Körperlänge haben. Die Beine stehen vom Körper frei hervor und sind als Schreitbeine sehr brauchbar. Wegen der langen umschlagbaren Klauen spielen sie auch als Klammerorgane eine große Rolle. Die Nachschieber sind sehr stark entwickelt und stehen frei hervor. Cber dem ganzen Körper, besonders am Kopfe ein wenig hinter den Augen, Pronotum und an den Abdominalsegmenten ragen lange steife Borsten hervor, die wahrscheinlich wenigstens teilweise im Dienste der Lokomotion stehen. In den Aquarien habe ich gesehen, daß die Larven sich sehr geschickt bewegen. Es ist daher wahrscheinlich, daß dieselben leicht selbst ihren Weg nach dem Wasser finden können.

Die hier mitgeteilten Beobachtungen stimmen übrigens gut mit dem, was Siltala in seiner schönen Arbeit 1906 b S. 47 bis 49 und S. 53 und a. a. O. berichtet hat; nichts streitet gegen Siltalas Beobachtungen, und diese sind vielleicht ein wenig erweitert worden. Ubrigens verweise ich auf seine Arbeit.

In den folgenden Stadien verschwinden alle diese Charaktere; bei den ausgewachsenen Larven (Fig. 28) liegen die zwei letzen Beinpaare viel mehr an den Körper angedrückt; sie dienen wie die ersten Beinpaare mehr als Klammerorgane, sind viel kürzer und erreichen nicht mehr als ${ }^{1} / 3$ der Körperlänge; die Klauen sind nicht so lang, und die Nachschieber sind kleiner und nicht so frei hervorstehend, auch die Borsten sind nicht so stark entwickelt.

Auf welchem Stadium alle diese Charaktere verloren gehen, weiß ich nicht mit Sicherheit zu sagen. Die verschiedenen Stadien Siltalas (1907a) 
sind, so lange sie nicht an die Häutungen angeknüpft werden können, meiner Meinung nach nur von ziemlich geringem Wert. Jedenfalls steht es fest, daß die vier ersten Stadien Siltalas sehr schnell durchlaufen werden. Im Sommer 1909 kamen wahrscheinlich keine Larven eher als ca. 15. .Juli hervor; schon am 10. Aug. sind alle Larven, die ich gesehen habe, im vierten Stadium (Länge 10 bis $17 \mathrm{~mm}$ ), und viele waren im fünften Stadium. Ich bin geneigt zu glauben, daß nicht jedem der vier Stadien Siltalas eine Häutung entspricht, und daß mehrere Häutungen zu seinem fünften und letzten Stadium gehören. Doch ist es mir unmöglich, hierüber etwas sicheres zu sagen.

Wenn die jungen Larven das Wasser erreichen, bauen sie in wenigen Stunden in meinen Aquarien ihr erstes Haus; ich habe hierzu den Tieren kleine Stücke von Halmen usw. angeboten; sie wurden gierig ergriffen und für den Bau der Köcher verwendet. Die Köcher waren unregelmäßig und interessierten mich nur so lange, bis ich die Larven des ersten Stadiums in der Natur gefunden hatte.

Als ich nun die Verhältnisse in der Natur studierte, fand ich eines Morgens Gallertmassen, die in ihrem Innern leere Eierschalen und keine Larven enthielten; in der nächsten Umgebung der Pflanzen (einer Iris) fand ich auf den submersen fein geteilten Blättern von Sium latifolium große Mengen grauer, langsam herumkriechender Staubpartikeln, die sich bald als Glyphotaeliuslarven erwiesen. Die Larven (Fig. 2) waren alle entweder in dem ersten oder zweiten Stadium Siltalas; keine über $4 \mathrm{~mm}$ lang und krochen geschickt auf der Vegetation umher.

Der Köcher, der nicht mehr als $3 \mathrm{~mm}$ messenden Larven ist ein kleines gallertartiges hyalines Rohr, nur ca. $4 \mathrm{~mm}$ lang und ca. $1 \mathrm{~mm}$ breit, in seinem hinteren Teil mit kleinen schwarzbraunen Wurzelresten bedeckt; diese liegen alle mit der Längsachse parallel. Wenn das Rohr nur 2 bis $3 \mathrm{~mm}$ lang geworden ist, nimmt das Tier ein Stück Halm, (Fig. 3 bis 5), das es wahrscheinlich abbeißt. Dieses Stück ist ca. 4 bis $6 \mathrm{~mm}$ lang und I bis $2 \mathrm{~mm}$ breit; es wird der Quere nach an das Rohr angebracht, immer am vorderen Teil und so, daß es über die vordere Röhrenmündung bedeutend hervorragt; auf diesem Stück baut das Tierchen nun die Seitenteile des Rohres weiter und fügt in dieselben ganz kleine Pflanzenpartikeln. Wenn die Seitenteile als zwei Wälle hervortreten, wird ein neues Querstück auf der entgegengesetzten Seite angebracht und immer so, dab der hintere Rand des neuen Stücks den vorderen des älteren, schon angebrachten überragt. Immer baut das Tier so, daß das Rohr genau in der Mitte des ganzen Köchers liegt, das heißt so, daß gleich große Teile über das Rohr hinausragen; dieses selbst hat nur eine Breite von einem Drittel des ganzen Köchers; es wird von dem Bauplan des Tieres bedingt, daß die zwei Breitflächen des Kööchers, für jedles neue eingefügte Querstück wechselweise 
Rücken- und Bauchseite ist. Die Querstücke werden mit dem "Boden" und dem "Deckel“ des Rohres zusammengewebt, im allgemeinen jedoch so, da $B$ nur die mittleren Teile mit dem Gespinste zusammenhängen, während die vorderen und hinteren Ränder frei hervorragen. Sobald das Tier 3 bis 4 Querstücke angebracht hat, geht das hintere unbedeckte Ende zugrunde; wahrscheinlich fällt es ab; möglicherweise wird es abgebissen. So fügt also das Tier wechselweise bald auf der „Rücken"- bald auf der „Bauchseite" ein neues Stück zu und baut an dasselbe die Seitenwälle des Rohres an.

Indem der Köcher an seinem vorderen Ende wächst, nimmt er in dem hinteren immer ab; so lange die Köcher noch wachsen, sind die Seiten schwach divergierend, später werden sie parallel. Eine besondere Verschlußmembran am Hinterende findet sich bei den Larven nicht; das Gespinst verschmälert sich hinten und wird so zusammengesponnen, daB nur ein kleines Loch, für den Ausstoß der Exkremente bestimmt, zurückbleibt. Im allgemeinen ist der Köcher beinahe zweimal so lang als die Larve; wird er aber zu einer Zeit gemessen, wo eine Spitze gerade lange nicht abgebrochen ist, kann das Rohr dreimal so lang werden. - So lange das Tier nicht über ea. 10 bis $17 \mathrm{~mm}$ ist (viertes Stadium Siltalas), bestehen die Querstücke gewöhnich aus abgewelkten Gras- und Carexhalmen und anderen Vegetabilien, die die Larven am Boden der Gewässer finden. Wenn sie ca. 14 bis $20 \mathrm{~mm}$, und die Köcher ca. 20 bis $30 \mathrm{~mm}$ erreicht haben, sind sie an den hier besprochenen Lokalitäten nur von einer einzigen Pflanze Potamogeton natans aufgebaut. In diesem Stadium verlassen nämlich die Tiere den Boden; sie steigen den Stengeln und Blattstielen des Potamogetons folgend bis an die Oberfläche, wo sie sich eine neue Heimat suchen. Das Aufsteigen findet in dem letzten Teil des August statt, und bald sieht man sowohl an der Größe der Köcher wie auch an den Potamogetonblättern, daß ein neues Leben begonnen hat.

Die Larven schneiden sich große elliptische Stücke aus den Blättern der Potamogeton (Fig. 6 bis 13); vom Rande ausgehend geht die Schneidefurche gegen die Mittelrippe hinein und erreicht nur selten dieselbe. Die Blattabschnitte werden beinahe immer so gemacht, daß der äußere Rand des Blattes einen größeren Teil des Abschnittes begrenzt, sie bekommen daher eine elliptische Form mit einem geraden und einem mehr oder weniger gebogenen Rand. Die Stücke werden am häufigsten so eingefügt, daß der gerade Rand sich gegen das hintere Ende wendet. Die Ränder sind immer sehr scharf abgeschnitten, wenn sie gefranzt sind, rührt es von späterer Verwesung her. Die Blattstücke der beiden Seiten sind so eingefügt, daß sie unter recht spitzem Winkel miteinander zusammenstoBen, so daB ein dreieckiger vom Wasser durchströmter Kanal an beiden Seiten des Rohres liegt (Fig. 25). 
Solange das Tier wächst und wahrscheinlich noch einige Zeit, nachdem das Wachstum aufgehört hat, werden immer neue Stücke an das vorderste angebracht und die alten am Hinterende abgeworfen. Dies geht daraus hervor, daß die Köcher bis Mitte Oktober immer größer werden, ohne daß jedoch die Anzahl der Stücke zunimmt. Weitere Beweise hierfür sind auch, sowohl daß die Oberflächen der Teiche mit zahlreichen abgeworfenen Blattstücken bedeckt sind, als auch daß die Blattstücke des Köchers mindestens bis September sich immer frisch grün erhalten.

Die größten Köcher, die ich gemessen habe, sind $55 \mathrm{~mm}$ lang und ca. 25 bis $30 \mathrm{~mm}$ breit. In den Herbstmonaten sieht man den Einfluß der Glyphotaelien an den Potamogetonblättern sehr deutlich. Der Angriff fängt vom Uferrande an, erreicht aber im Oktober den äußersten Rand der Potamogetonzone. Im Jahre 1908 war die ganze Zone beinahe abgeblättert, nur die leeren Blattstiele und die Rudimente der Blätter waren übrig geblieben. Daß die Larven der Hydrocampen, die nicht häufig waren, zu diesem für die Potamogetonpflanzen traurigen Resultate beigetragen hatten, ist unzweifelhaft; ihre Hauptentwicklungsperiode fällt aber in die Monate Juni und Juli, nicht wie die der Glyphtotaelien in den Spätherbst. Es war im Oktober merkwürdig, die tausende und abertausende von Blättern der Pflanzen abgelöst zu sehen. und an den Köchern der Glyphotaelien wieder zu finden. Die Potamogeton mußten nicht allein Material für die Wohnung der Tiere abgeben; sie waren zugleich deren Nahrungspflanze; die Unterseite der Blätter wurde abgenagt, die Oberhaut der Oberseite im allgemeinen geschont. Der Darmkanal der Larven war mit dem grünen breiartigen Material vollgepfropft, und als der Angriff am stärksten war, sah man überall die braungrünen Exkremente an den Blattstielen oder langsam durch das Wasser fallen. Meistens hängt das Tier mit den Beinen an den Stielen der Potamogeton zirka $1 \mathrm{~cm}$ unter dem Wasserspiegel befestigt; doch oft findet man auch die Köcher mit einer der Breitflächen nach oben frei zwischen den Potamogetonblättern herumschwimmen; schlägt man mit den Rudern in die Potamogeton, so verlieren die Tiere ihre Anheftungsstellen und schwimmen dann an oder direkt unter der Oberfläche herum; einzelne sinken aber außerordentlich langsam und stoßen gewöhnlich auf die Potamogetonstengel, ergreifen dieselben und klettern wieder in die Höhe. Die Tiere selbst sind übrigens sehr lebhaft, wenn sie an der Oberfläche in der kleinen pelagischen Region zwischen den Potamogetonblättern liegen, strecken sie den Vorderkörper sehr lang aus dem Köcher hervor und schlängeln denselben bald nach rechts bald nach links; dadurch rudern sie sich durch das Wasser nahe an der Oberfläche und erreichen bald ein anderes Blatt, an welchem sie sich festhaken. Oft sieht man die Larven Luft abgeben; die Luft liegt in den Röhren; wahrscheinlich rührt sie von den Narben der frisch abgebissenen Pflanzenteile, wovon 
der Köcher bedeckt ist, her; es ist aber auch möglich, daß die Tiere, wenn sie sich an der Oberfläche aufhalten, und den Körper schlängelnd außerhalb des Rohres bewegen, Luft zwischen Körper und Rohr bekommen. Daß diese Luft für die Tiere, so lange sie ihre Nahrung in den Schwimmblättern suchen, von Bedeutung ist, versteht sich von selbst. Hierauf kommen wir später zurück.

Am Schlusse des September bemerkt man, daß die Röhren nicht mehr die frische grüne Farbe besitzen; die Blattstücke sind gelbgrün und tragen Spuren von Vermoderungsprozessen, gleichzeitig beginnen auch die Blätter der Potamogetonzone abzuwelken.

1907 im Oktober, 1908 aber erst im November, sieht man, daß die äußere Form der Köcher sich ändert; sie ist nun nie so breit; gewöhnlich nur ca. $20 \mathrm{~mm}$, bleibt aber immer ca. $50 \mathrm{~mm}$ lang. Die Deckstücke sind kleiner und bestehen nicht ausschlieBlich aus Potamogeton; oft rühren sie von abgewelkten Erlenblättern her, die von den Erlen am Ufer auf die Seeoberfläche niedergefallen sind. Zugleich findet man Köcher, die in ihren hinteren Teilen noch von großen Potamogetonblättern bedeckt sind, während solche im vorderen Teile ganz fehlen. Hier entbehrt das Rohr alle deckenden Querstücke und besteht aus grünen Stücken von Blattstielen der Potamogeton (Fig. 14 bis 17). Zugleich nimmt das Tier ein ganz neues Bauprinzip an; indem diese Stücke nun nicht quer, sondern parallel der Längsachse des Rohres angebracht sind. Indem die Tiere von November bis Ende Dezember dieses neue Material nach einem neuen Bauprinzip anwenden, entstehen Köcher von einer von den bisherigen ganz. verschiedenen Form. Die Köcher werden röhrenförmig und gleichen flüchtig gesehen den Köchern von Phryganea grandis, weichen aber von diesen dadurch $a b$, daß die Stielstücke nicht gleich lang sind; in mehreren Röhren glaubt man sogar eine schraubige Anordnung der Stücke zu finden. Man findet im November alle mögliche Ubergänge (Fig. 17 bis 18) zwischen den breiten, aus Blättern und den röhrenförmigen aus Stielstücken gebauten Köchern. Es geht aus den Übergangsstadien hervor, daß die Tiere erst ca. $30 \mathrm{~mm}$ lange Blattstiele abbeißen und über den hinteren Deckstücken parallel mit der Längsachse des Rohres anbringen; die Stiele sind daher in der ganzen Länge dem Rohre angeklebt, die späteren nur mit dem vorderen Ende; mit dem hinteren stehen sie wie grobe Bürsten ab.

Das Material, auf dem diese Mitteilungen beruhen, rührt vom 5. bis 20. November 1908 her. Als ich am 26. November die Teiche wieder besuchte, waren sie zugefroren. Das Eis wurde eingebrochen, und es zeigte sich, dab die Glyphotaelien sich noch in der Potamogetonzone befanden. Am 1. Dezember taute der Teich wieder auf, während des Auftauens des Eises vermoderten die Blätter der Potamogeton vollständig, und sehr viele wurden langsam auf dem Boden abgelagert. Noch saßen aber die Glypho- 
taelien in großer Menge auf den Blattstielen, die noch grün waren. Am 26. Dezember bekamen wir plötzlich Schneesturm; der Teich fror wieder zu, wurde aber im Gefrierstadium vom Boot aus beobachtet; die Potamogetonregion war nun beinahe von Glyphotaelien frei, wenn man aber nit einem Stangnetze von ca $2 \mathrm{~m}$ den Boden untersuchte, fand man ca. $11 / 2 \mathrm{~m}$ bis $1 / 2 \mathrm{~m}$ vom Ufer zahlreiche Glyphotaelien. In den Tagen vom 26. Dezember bis 28. Dezember fror der Teich zu und war bis 15. April zugefroren.

Die vom 26. Dezember herührenden Glyphotaelienköcher (Fig. 19 bis 20) hatten noch die obengenannte, zylindrische Form und waren hauptsächsich aus Potamogetonstielstücken gebaut.

Wie schon oben bemerkt, geht der Übergang von den breiten Röhren mit Querstücken zu den schmalen mit nach der Längsachse geordneten Stücken im allgemeinen ganz langsam vor sich. Eben in den letzten Tagen des Dezember, so wie auch im April, nach dem Auftauen, fand ich auf dem Boden des Wassers sehr oft schöne, von Blättern gebaute Röhren, die aber alle leer waren. Ich bin daher geneigt zu glauben, dab die Tiere, deren Röhren Ende Dezember noch mit Querstücken von Blättern bedeckt sind, diese Röhren verlassen und sich neue bauen. Um so mehr glaube ich, daß dies richtig sein kann, weil solche Röhren nach dem Auftauen des Teiches im April nie mehr zu finden sind.

Wenn die Tiere also Ende Oktober bis November sich nach dem Boden begeben, sind sie graugrün, die schöne grüne Farbe des Hochsommers besitzen sie nicht. Der Darm ist mit Futter vollgepfropft, und eine Verschiedenheit zwischen dem Darminhalt im August und im Dezember ist schwer festzustellen, die Futterpflanze ist in der ganzen Zeit beinahe nur Potamogeton gewesen; es ist wohl möglich, daß die Tiere sich im November und Dezember vielmehr von Stengeln als von Blättern nähren.

Vom 26. Dezember bis 15. April ist der Teich fest gefroren. Am 27. Februar, als das Eis ca. $1 / \mathrm{m}$ dick ist, wird es von einem Stück des Ofers entfernt; unter dem Eise auf ca. $3 / 4 \mathrm{~m}$ sehe ich 10 Glyphotaelien auf dem Boden liegen oder langsam herumkriechen. Die Röhren (Fig. 21 bis 22) sind sehr interessant; an den Seiten oder vom Hinterende hinausragend, liegen noch die Potamogetonstiele, die Ober- und Unterseite des Rohres ist aber aus toten Pflanzenmaterialien, abgewelkten Blättern der Sparganien, der Carexarten und sedimentierten Erlenblättern aufgebaut. Alte Stücke sind parallel der Längsachse des Rohres geordnet und mit den Seiten des Rohres fest zusammengesponnen. Daneben findet man aber Röhren, die in ihrem hinteren Ende breite flügelförmig weit über die Seiten des Rohres hinausragende Erlenblätter besitzen. Auch solche mit Potamogetonblättern am hinteren Ende kann man beobachten; diese sind aber so vermodert, daß sie nicht breiter als das Rohr sind. 
Am 6. April war der Teich noch fest zugefroren, nur an dem nach Süden gelegenen Ufer hatte die Sonne das Eis spröde gemacht; hier und später am 15. April (Fig. 23), als der Teich noch zugefroren war, sah ich an dem Südufer auf dem Boden zahlreiche Glyphtotaelienköcher. Sie haben hauptsächlich dasselbe Aussehen wie am 27. Februar; näher besichtigt zeigt es sich jedoch, daß kein einziges Rohr mehr hervorragende quergeordnete Blattstücke besitzt, ferner, daB die Ober- und Unterseite des Rohres nur je 1 bis 2 größere oblonge Stücke tragen; nimmt man diese $\mathrm{ab}$, liegen die seidenglänzende Ober- und Unterseite ganz frei; an den Seiten sind die Kleinstücke so in Seide eingewebt, daß es beinahe unmöglich ist, sie abzunehmen.

Der Darmkanal der Tiere enthält im Februar wie im April immer Pflanzenteile; diese sind wie im Sommer nicht mehr grün, sondern braungelb und bestehen aus abgestorbenen Pflanzenteilen; oft findet man in dem Magen zugleich Algenreste, Dauerkeime und Eier von Bryozoen, d. h. Detritus, der sich teils am Boden, teils auf den Schilfstengeln gesammelt hat. Die Farbe der Larven ist nicht mehr graugrün, sondern gelblich weiß.

Der Darm nimmt kaum einen so großen Teil des Körpers ein, wie früher, dagegen ist der Fettkörper nun sehr dick, und er gibt der Larve ihre weißgelbe Farbe. Die Larven mit ihren Köchern sind nun schwere Organismen, die, wenn man sie ins Wasser wirft, augenblicklich zu Boden fallen; sie bewegen sich langsam und ungeschickt, und die Bewegung zwischen den Körperringen ist sehr klein.

Am 4. Mai, als ich den Teich wieder besuchte, findet sich beinahe keine Larve am Boden des Wassers mehr; zweifellos hat das Tier sich verpuppt, aber wo? Nach stundenlanger Untersuchung ohne Resultat, fand jch endlich etwas, das wie zusammengesponnene Carexblätter (Fig. 24), iuussah. Es war das Puppengehäuse, aber in einer völlig verschiedenen Gestalt von dem, wie ich es mir gedacht hatte. Die Puppengehäuse sitzen hauptsächlich auf den Carexblättern, nur ca. 1 dem unter dem Wasserspiegel. Sie waren schwer zu beobachten; am besten fand man sie, wenn man mit den Händen die Carexhalmen umfaßte; dann merkte man bald die dicken harten Körper. Noch war das ganze Larvenmaterial nicht verpuppt; einzelne krochen langsam umher.

Wenn die Larve sich zur Verpuppung anschickt, hängt sie sich an einem Carexhalm auf; sie nimmt dann von den herabhängenden welken braungelben Blättern erst kleine und dann später längere bis ca. $70 \mathrm{~mm}$ große Stücke, und spinnt sie in mehreren Schichten übereinander; nach und nach wird der ganze Köcher dicker. Die 1 bis $2 \mathrm{~cm}$ breiten Blätter werden in ihrer ganzen Länge dem Köcher fest angesponnen; da num die Blätter viel länger sind, als das primäre Gehäuse, ragen sie an beiden Enden ca. 10 bis $20 \mathrm{~mm}$ über dieses heraus; hier stoßen die Spitzen der 


\section{- $102-$}

aufgesponnenen Blätter zusammen, so daß das ganze Gehäuse eine spindelförmige Form bekommt.

Wenn das Puppengehäuse fertig ist, stellt die Larve noch die Siebmembranen (Fig. 30 bis 31) an beiden Enden des Rohres her; ihr gegenseitiger Abstand ist nur ca, $27 \mathrm{~mm}$ : diese Siebmembranen sind wahre Meisterwerke; sie sind mit den Blättern fest zusammengesponnen, und das Gespinst des Randes der Membran geht kravattenförmig an den Blättern hinauf. Sie bestehen aus einem Balkengerüst, dessen Zwischenräume mit gelber Seide ausgefüllt sind, nur in den innersten Winkeln, wo die Balken zusammenstoßen, sind Löcher zurückgeblieben. Die vordere Siebmembran ist immer regelmäBiger gebaut und hat nicht so viele Löcher (ca. 20) als die hintere (30 bis 40). Diese Siebmembranen findet man in großen Mengen in Torfablagerungen des kleinen Sees; dieselben oder gleicharlige sind bekanntlich allgemeine Fossilien in den alten quartären Torfmooren; dort liegen sie als beinahe einzig bestimmbares Material von dem Phryganeenleben der Vorzeit.

Wenn die Larve die Siebmembran spinnt, enthält der Darm noch recht bedeutende Menge von Nahrung, und in der Puppenwiege dauert das ruhende Larvenstadium noch einige Zeit; die Haut der nun beinahe ganz weißen Larven birst in der Rückenhaut des Kopfes und den zwei ersten Thorakalringen, und die Puppe kriecht aus. Puppenlänge ca. $20 \mathrm{~mm}$, also ca. $10 \mathrm{~mm}$ weniger als die innere Länge des Köchers.

In den Aquarien habe ich sehr oft viele Puppengehäuse gehabt; vergebens versuchte ich ausfindig zu machen, wie die Puppe ihre Putzapparate benutzt, weder an der vorderen noch an der hinteren Siebmembran habe ich einen Durchstoß des Putzapparates gesehen (cfr. Thienemann 1905 S. 1). Die Halme hängen über die Siebmembranen heraus und bewirken, daß man nichts sehen kann; nimmt man die Halme weg, verhält die Puppe sich vollkommen ruhig.

In der Natur fand ich die ersten Puppengehäuse am 4. Mai, und die Verpuppung hat wahrscheinlich nicht früher als am 1. Mai begonnen. Am 14. Mai sind wahrscheinlich alle verpuppt gewesen, am 27. Mai schwammen die ersten Puppenhäute auf der Oberfläche des Wassers, am 9. Juni in großen Mengen, und die Tiere saßen an der Ufervegetation. Der Aufenthalt im Puppengehäuse dauert wahrscheinlich ca. 2 bis 3 Wochen. Die ausgefärbte Puppe (Fig. 29) mit ihrem schön grüngefärbten Hinterleib, den schwarzen Seitenlinien und den rot gefärbten Flügeln ist ein sehr schönes Tier.

\section{Beobachtungen über das Puppenstadium der Wasserinsekten.}

Lange ehe ich mich mit Glyphotaelius beschäftigte, hat mich das freilebende Puppenstadium der Phryganeen interessiert. In meinen Aquarien habe ich von vielen verschiedenen Phryganeen, deren Namen mir damals 
wie jetzt unbekannt sind, freilebende Puppen zu hunderten gehabt. Ich erlaube mir hier einige dieser alten Beobachtungen einzuschieben.

Das freilebende Puppenstadium der Phryganeen ist sehr wenig bekannt; viele wissen gar nicht, daß ein solches existiert, und in den Lehrbüchern findet man nichts hierüber.

In meinem Aquarium dauert es höchstens 24 Stunden; waren die Puppen vor dieser Zeit nicht entwickelt starben sie aus. Unter natürlichen Verhältnissen dauert das freilebende Puppenstadium wahrscheinlich nicht so viele Minuten, es hat keine andere Bedeutung als das Tier aus dem Wasser zu bringen, so daß die letzte Verwandlung entweder an der Oberfläche des Wassers oder am Lande vor sich gehen kann.

Auf dem Boden meiner Aquarien habe ich oft die ausgekrochenen Puppen auf den Gehäusen sitzen sehen. Plötzlich hebt das Tier sich; das erste Beinpaar ist gewöhnlich vorwärts gestreckt, das dritte Beinpaar wird parallel dem Körper vollkommen ruhig gehalten, und nur mit dem zweiten Beinpaar schwimmt das Tier. Dieses Beinpaar ist bekanntlich mit langen, schwarzen Haarfranzen auf beiden Seiten der Tarsen und oft auf den Schienen besetzt. Die Mittelbeine werden gleichzeitig und in großen Bogen, wie die Hinterbeine der Notonecten bewegt. Wenn die Tiere sich ruhig verhalten, stehen die Mittelbeine beinahe senkrecht mit großen Winkeln zwischen femora und tibia vom Körper ab. Die Schwimmbewegungen dauern nur kurze Zeit, nicht über 5 Minuten, dann sinkt das Tier wieder zu Boden; was sie am meisten charakterisiert, ist die Plötzlichkeit womit sie beginnen und wieder aufhören, ferner die erstaunliche Schnelligkeit und große Kraft in den einzelnen Stößen, wobei die Bewegung durch das Wasser eine hüpfende wird. Falls es dem Tiere in den obgenannten 5 Minuten nicht gelingt, entweder feste Gegenstände, die auBerhalb des Wassers hervorragen oder die Oberfläche selbst zu erreichen, sinkt es wieder zu Boden. Nach einem kleinen Ausruhen wird ein neuer Versuch gemacht, und wenn das Ziel im Laufe von ca. 12 bis 24 Stunden nicht erreicht ist, verweilt das Tier auf dem Boden und stirbt da ab. So lange es ruhig sitzt, schlägt der Hinterkörper regelmäßig auf und nieder, ca. 30 Schläge in der Minute (Atmungsbewegungen).

In zahlreichen Fällen habe ich gesehen, daß das Tier unmittelbar, nachdem es ausgekrochen war, auf dieselben Grashalme, dio es als Befestigungsblatt seiner Puppenwiege gebraucht hatte, hinaufkroch, die Wasseroberfläche erreichte und wenige $\mathrm{cm}$ über derselben sich verwandelte; hier gebrauchte das Tier also gar nicht sein Schwimmvermögen. Oft findet man, daß die Larven sich nur wenige $\mathrm{cm}$ unter dem Wasserspiegel verpuppen, und $\mathrm{d} a \mathrm{~B}$ die Puppengehäuse, wenn eine Trockenperiode eintritt und die Teiche sehr seicht sind, über das Wasser zu hängen kommen. Dies braucht nicht der weiteren Entwicklung zu schaden, und auch in diesen Fällen 
haben die ausgekrochenen Puppen keinen Vorteil von ihrem Schwimmvermögen.

Diese Beobachtungen beziehen sich wahrscheinlich alle auf Limnophiliden; näher bestimmt wurden die Tiere leider nicht. Mit den Leptoceriden verhält es sich anders. Arn Morgen des 14. Juni 1891 Uhr 8,40 sah ich eine Leptoceride aus dem Puppenköcher hervorbrechen; sie schwamm ca. 4 Minuten herum, erreichte die Oberfläche und lag dann eine Minute ruhig da, indem sie die Seitenlinie und Tracheen als Anheftungsapparate an die Wasseroberfläche brauchte: die ganze Rückenfläche, die oberhalb des Wassers lag, war trocken, und sah fettartig aus. Schwimmend in der Oberfläche machte das Tier ein paar schnelle Bewegungen mit den Mittelbeinen, dann faltete die Haut des Kopfes und der Brust sich plötzlich auswärts, wodurch eine flache Schwimmscheibe entstand; zugleich wurden die Flügelscheiden auswärts geschlagen und stellten sich in das Wasser tauchend vertikal unter dem Abdomen ein. Die Tarsen der Vorder- und Mittelbeine traten augenblicklich auf die Wasseroberfläche, das Tier zog dann die Hinterbeine heraus, die sich auf die Schwimmscheibe stellten. Dann zog es von unten nach oben die Flügel aus, und in derselben Minute flog es an mein Fenster das ganze dauerte nur ca. 2 Minuten; mehrmals und bei verschiedenen Leptoceriden habe ich dasselbe beobachtet; es scheint, als ob jedenfalls mehrere derselben wie die Mücken sich an der Oberfläche befestigen, und daß die letzte Mauser eben hier vor sich geht. Ich habe auch Limnophiliden in Aquarien stundenlang an der Oberfläche hängend sehen; doch habe ich niemals Tiere in dieser Stellung auskriechen sehen. - Uber die Familie der Phryganidae u. a. habe ich leider keine Beobachtungen.

Viel später als ich 1906 Siltalas schöne Untersuchungen bekam, sah ich, daB unsere Beobachtungen (1906a S. 88) gut miteinander übereinstimmten.

Wie schon oben bemerkt, hat das freilebende Puppenstadium keine undere Bedeutung, als das Tier so schnell wie möglich aus dem Wasser zu bringen. In vielen Fällen machte es gar keinen Gebrauch von seinem Schwimmvermögen oder die Schläge, die es mit den Mittelbeinen ausführt, beschränken sich auf ganz wenige. Was mich hier am meisten interessiert, ist zu sehen, in wie hohem Grade diese wenigen Minuten im Leben der Puppen ihre ganze Organisation so zu sagen geprägt haben, teils neue Organe ausgebildet, teils alte modifiziert haben. Ich denke hier an die Entwicklung von Schwimmhaaren an den Mittelbeinen besonders den Tarsen; bei einigen Phryganeen kommen auch solche an den Vorderbeinen vor, sind aber hier nur selten so stark entwickelt; die Hinterbeine sind beinahe ganz kahl. Wir haben es hier mit einem Bewegungsapparat zu tun, der vielleicht nie gebraucht werden soll; höchstens dann und wann in einer Zeit von 24 Stunden und in vielen, wahrscheinlich in den meisten 
Fällen, nur während weniger Minuten; man muß annehmen, daß die Tiere gewöhnlich den Bewegungsapparat nicht zu mehr als ein paar ganz vereinzelten Schlägen brauchen. Ein ererbter Apparat ist es durchaus nicht, weil überhaupt - meines Wissens - kein einziges anderes Insekt die Mittelbeine als Sehwimmbeine allein und in dieser Weise braucht; es ist ein für die Phryganiden spezifisches Bewegungsorgan. Wie kurz auch die Zeit war, in der es gebraucht werden sollte, seine Herstellung war dennoch eine Lebensbedingung für das Tier, und das Organ wurde hervorgebracht: wie und wann, das sind Fragen, die ich vorläufig den Descendenzttheoretikern überlasse; doch hoffe ich, daß ich hierauf später zurückkommen kann.

Die Puppen haben auch andere Organe, die in dieser kurzen Zeit des Lebens und eben nur in dieser eine Rolle spielen. ${ }^{1}$ ) Bekanntlich ist die Rückenfläche des Abdomen der Puppe mit besondern Haftapparaten versehen. Der erste Hinterleibsring trägt entweder zwei mit feinen Spitzen bedeckte Warzen oder eine sattelförmige Erhöhung; das 3. bis 7. Segment trägt auf der Rückseite ein paar Chitinplättchen mit Haken und das כ. Segment ferner ein paar größere Chitinplättchen. Man sagt oft, daß dieselben in dem Dienste der Verschiebungsbewegungen stehen. Doch haben diese Organe sicherlich auch eine andere Funktion, und dies ist wahrscheinlich ihre Hautfunktion.

Wir finden ganz gleichartige Chitinbildungen bei Puppen einer anderen Insektenordnung, wo die Puppen auch dazu bestimmt sind an der Oberfläche horizontal zu hängen und sich dort zu verwandeln. Ich denke hier an die Puppen vieler Tipuliden. Alle diese Chitinbildungen sind Organe, mittelst welcher die Tiere die Wasseroberfläche brechen und dann an der Oberfläche hängen. Wer die an der Oberfläche hängenden Phryganeen und Tipulideupuppen (z. B. Phalarocera) beobachtet hat, kann sich davon überzeugen. Aber auch die Seitenlinie, die bei den Puppen besonders stark entwickelt ist und einen in seinem hinteren Teil mehr als bei der Larve ventralwärts gebogenen Verlauf hat ist ein Mittel, durch welches die Larve auf der Oberfläche ruht. Ich bin daher geneigt, sowohl in den obengenannten Chitinfortsätzen als in der starken Entwicklung der Seitenlinie Mittel zu sehen, womit die Puppen sich an der Oberfläche halten,

1) Von den eigentümlich geformten Puppenmandibeln, die keine andere Rolle spielen, als die Seitenstränge der Verschlußmembran durchzuschneiden, und die von den Larvenmandibeln ganz verschieden sind, selie ich hier ab. Das eigentümliche des Anftretens dieses Organs bei den Puppen wird dadurch erheblich gestärkt, daß die Trichopteren als imagines bekanntlich entweder keine oder sehr rudimentäre Mandibeln besitzen. Was gebraucht werden soll, wird hervorgebracht; was nicht, wird in der Rumpelkammer der Spezies aufmagazinicrt; später in der Zukunft der Spezies liann es wieder herausgenommen, nm in vielleicht modifiziorter Gestalt nochmols angewandt zu werden. 
und zu glauben, daß diese Funktion eine Lrsache zu ihrer Bildung gewesen ist.

Die Natur hat augenscheinlich alle den Wasserinsekten, die ihre Puppen- oder Nymphenstadien im Wasser zubringen, eine schwere Aufgabe gestellt; mit unbenetzten Flügeln sollen sie sich aus dem feuchten Elemente direkt in die Luft hinaufschwingen können. Zugleich soll ein für das Wasserleben angepaßtes Respirationsorgan mit einem für das Luftleben angepaßten vertauscht werden. Die Anfgabe ist bei den verschiedenen Insektenordnungen auf verschiedene Weise gelöst. Lm das frei lebende Puppenstadium der Phryganeen besser zu verstehen, sei es mir erlaubt, kurz diese verschiedenen Modi zu erwähnen. Wir finden bei vielen der wasserbewohnenden Dipteren, besonders den meisten Mücken Verhältnisse, die sehr mit denen der Leptoceriden übereinstimmen; auch viele Ephemeriden verhalten sich in derselben Weise; doch finden wir hier zwischen dem Wasser und Luft bewohnenden Stadium das merkwürdige, einzig dastehende Subimagostadium eingeschaltet. Die Odonaten kriechen, wenn sie sich verwandeln sollen, aus dem Wasser heraus und verwandeln sich in der Luft. Doch auch hier gibt es ein merkwürdiges wenig beachtetes Zwischenstadium, das nicht ohne Interesse ist. Bekanntlich öfnen sich im letzten Nymphenstadium die großen Prothorakalstigmen. Ehe die Tiere direkt in die Luft hinauf kriechen, sitzen sie mehrere Stunden nur mit dem Kopfe und dem ersten Prothoraxring oberhalb des Wassers. In dieser Stellung atmen sie die atmosphärische Luft durch die Prothorakalstigmen noch vom Wasser benetzt und verlassen erst später dieses vollständig. Wenn man erst einmal auf dieses Phänomen aufmerksam geworden ist, kann man an hellen Frühsommermorgen hunderte von Aeschnen in dieser Stellung an den Grashalmen und gerade in der Wasseroberfläche sitzen sehen.

Was die Schmetterlinge des SüBwassers anbelangt, gleichen sie in ihren biologischen Verhältnissen den Phryganeen sehr; dies gilt vielleicht besonders von Hydrocampa nymphaeata. Ihre aus zwei Blättern zusammengesetzten Puppengehäuse sind an Wasserpflanzen befestigt. Die Larven beißen einige Löcher in dieselben und fangen die davon ausströmende Luft in einem Gespinst, womit sie die Innenseite der zwei Blätter auskleiden, auf; etwas gleichartiges finden wir bei den Donacien. Dann verpuppt das Tier sich. Die Puppe ist eine wahre Schmetterlingspuppe ohne Bewegungsvermögen und vermag nicht wie die Phryganeenpuppen durch die wenige Zentimeter dicke Wasserschicht, die sie von der Luft scheidet, zu schwimmen. Wie kann hier die Verwandlung vom Wasserinsekt zum Luftinsekt vor sich gehon? In den letzten Tagen des Puppenlebens nimmt die Puppenhaut eine fast goldene Farbe an, ein Phänomen, das man auch bei Fliegenpuppen, die in Wasserpflanzen leben, 
beobachten kann. Dies rührt von Luft, die sich unter der braunen Puppenhaut um die Puppe sammelt, her. Wenn nun das Tier das Puppengehäuse verlassen soll, wird diese Luft von den Flügeln gefangen und das Tier von der Luftkugel blitzschnell durch das Wasser getrieben. Die Flügel sind, wenn sie aus der Puppenhaut herausgezogen werden, mit einem weißen Wachsüberzug bedeckt, und während der blitzschnellen Fahrt durch das Wasser gleitet der Wachsüberzug ab. Daher sieht man einige Minuten, nachdem das Tier die Oberfläche erreicht hat, den senkrechten Weg von der Puppenwiege bis an die Oberfläche als eine weiße, silberglänzende Säule oder einen Strang markiert; schwebend steht die Säule einige Minuten, die feinen Staubpartikel gleiten auseinander und verschwinden. In demselben Augenblicke, wo das Tier die Oberfläche erreicht hat, läuft es schnell wie eine Phryganide über die Wasseroberfläche bis an das Ufer, wo das volle Entfalten der Flügel vor sich geht. Die hier mitgeteilten Beobachtungen rühren von Aquarienstudien vom 15. Juni 1891 her. Ich habe jenes momentane AusschieBen der Puppe dreimal beobachtet. Wie sich Paraponyx und Acentropus verhalten, weiß ich leider nicht; Cataclysta befestigt ihre Puppengehäuse meist oberhalb des Wassers.

Die obengenannten Aufgaben werden also in recht verschiedener Weise gelöst; in den verschiedenen Insektenordnungen finden wir an der Schwelle zwischen dem Wasser- und Luftleben eigentümliche biologische Verhältnisse, die mehr oder weniger die Organisation der Insekten prägen. Das freilebende, nur wenige Minuten, höchstens einige Stunden dauernde Puppenstadium der Phryganeen mit seinen eigentümlichen, eben für dieses kurze Lebensintervall eingerichteten hoch spezialisierten Bauverhältnissen ist in dieser Weise aufzufassen und soll mit den entsprechenden obengenannten Stadien und eigentümlichen Strukturverhältnissen bei anderen Insekten verglichen werden.

Wir kehren nun nach diesem langen Ausschweifen wieder zu Glyphotaelius zurück. Wenn das Tier ausgekrochen ist, ist es noch nicht ganz ausgefärbt; dies geschieht, indem es auf den Grashalmen nahe am Ufer ruht. Ich sah die ersten Glyphotaelien am 27. Mai (Fig. 32), die letzten am 15. Juli. Ihre Lebensdauer ist wahrscheinlich recht kurz. Die Zeit, in welcher die Eiablage vor sich geht, ist wahrscheinlich ziemlich lang. Leider sah ich nie eine Paarung. Das Tier war wenigstens bei Tage merkwürdig selten. An einem Sommerabend sah ich ziemlich viele über die Ufervegetation fliegen, doch nie in solchen Mengen wie die Hydrocampen, die an einzelnen Tagen wie Schneegestöber über dem Wasserspiegel standen. Nahrungsaufnahme der Imagines sah ich nicht, und auf den Blumen waren sie wenigstens am Tage nicht zu finden; weil 
aber die Teiche weit entfernt von meiner Heimat waren, habe ich nur wenige Abendbeobachtungen. Am Tage sitzen die Tiere immer vollkommen ruhig geschützt im Grase des Ufers.

Es scheint, als ob die Glyphotaelien nur wenige Feinde haben; in den Eimassen sah ich nie Schmarotzer; ich suchte solche um so eifriger, als ich in anderen Limnophilideneimassen mehrmals Fliegenlarven gefunden habe. Ich besitze Eierhaufen von Limnophiliden, die ca. $1 \mathrm{~m}$ über dem Wasser abgesetzt sind ("tropfende Eiermassen") und worin 2 bis 3 weißgelbe, $1 \frac{1}{2} \mathrm{~cm}$ lange Fliegenlarven liegen. Mehrmals sah ich Puppenköcher, die auf einer der Seiten ein Loch von ca. $1 / 2 \mathrm{~cm}$ Durchmesser hatten; aus solchen Löchern krochen immer Nepheliden uns; inwieweit sie die Löcher gebohrt haben, weiß ich nicht; in solchen Köchern fanden sich immer nur Reste von Puppen.

Wir haben nun die Biologie der Glyphotaelien auf dieser kleinen nordseeländischen Lokalität verfolgt. Auf meinen zahlreichen Exkursionen habe ich oft die Glyphotaelien gesehen, nie aber in solchen Mengen wie in meinem Versuchsteich. Nur selten fanden sie sich an der Oberfläche und dann nur da, wo diese beinahe von Blättern, besonders Potamogeton bedeckt war. Oft war das Gehäuse von anderen Blättern als denen des Potamogeton gebaut, immer wurden jedoch bis Oktober grüne Blattstücke gebraucht, und der Bauplan des Köchers war stets derselbe. Im Gripsee waren die Köcher von Erlen- und Birkenblättern gebaut, und hier krochen die Juarven immer auf den Fontinalisteppichen des Bodens; in dem Funkenteich wurden besonders Teile von Nymphaeaceen gebraucht.

In der Iiteratur sind die Glyphotaelien oft erwähnt, besonders bei De Geer $(1778$, p. 368$)$, Struck $(1899$, p. 1) und Siltala, auf deren Arbeiten ich hinweise. Besonders bei den letztgenannten $(1902$, p. 39) findet man eingehende Schilderungen von den Bau der Larven und Puppen. Keine der biologischen Beobachtungen steht in fundamentalem Gegensatz zu den meinigen, und diese haben die früheren hoffentlich ergänzt und erweitert.

Wir können nun die Hauptpunkte der Biologie von Glyphotaelius punctatolineatus im Versuchsteich II folgendermaßen rekapitulieren.

\begin{tabular}{|c|c|c|}
\hline \multirow{2}{*}{$\begin{array}{l}\text { Eierstadium } \\
\text { Lauvenstadium am Boden des Gewässers }\end{array}$} & ca. 15. Juni-1. Aug. & \\
\hline & 1. Aug. - 1. Sept. & $\begin{array}{l}\text { Kōcher: quergelegte Deck- } \\
\text { stücke abgestorbener Blatt- } \\
\text { stücke. }\end{array}$ \\
\hline an Potamogeton & 1. Sept. - 1. Nov. & $\begin{array}{l}\text { Köcher: quergelegte Deck- } \\
\text { stücke yon lebenden Pflan- } \\
\text { zen (Blätter von Potam.). }\end{array}$ \\
\hline , an Potamogeton & 1. Nov, - 1. Dez. & $\begin{array}{l}\text { Köcher von longitudinal ge- } \\
\text { legten Deckstucken (Sticle } \\
\text { von Potam.). }\end{array}$ \\
\hline
\end{tabular}




\begin{tabular}{|c|c|c|}
\hline Larvenstadium am Boden des Gewässers & 1. Dez. - 1. Jan. & $\begin{array}{l}\text { Köcher von longitudinal gc- } \\
\text { legten Deckstücken (Stiele } \\
\text { von Potam.). }\end{array}$ \\
\hline$" \quad$ am Boden des Gewässers & 1. Jan.-1. Mai & $\begin{array}{l}\text { Köcher von longitudinal ge- } \\
\text { ordnetem Bodenmaterial. }\end{array}$ \\
\hline Puppenstadium & 1. Mai - 1. Juni & \\
\hline Imago & ca. 1.Juni - 1. Juli $\left.{ }^{1}\right)$ & \\
\hline
\end{tabular}

\section{Theoretisehe Betrachtungen und Versuche.}

Was in dieser kleinen Untersuchung am meisten interessiert, sind natürlich die verschiedenen Köcher.

Bekanntlich ändern viele Phryganeenlarven im Laufe ihrer Lebenszeit die Baustoffe der Köcher; dagegen wird nur selten die primäre röhrenförmige Gestalt des Köchers geändert. Wir haben gesehen, daß die jungen eben ausgekrochenen Glyphotalelien beinahe augenblicklich die Köcher viel breiter als das Rohr machen, ferner daB sie später als Oberflächentiere damit eine Zeit fortfahren, dann im November eine neue Bauart mit longitudinal gelegten Pflanzenteilen annehmen und damit fortfahren bis zur Verpuppung.

Warum fangen sie diese merkwürdige Bauart an, und warum verlassen sie sie wieder? Um diese Frage zu lösen, arbeitete ich zuerst viel mit Aquarienstudien, doch gaben sie alle kein befriedigendes Resultat. Dann richtete ich wie früher so oft meine Versuche in der Natur selbst an. An den klaren schönen September- bis Oktober-Tagen studierte ich von meinem Boot aus in der Potamogetonregion von hunderten von Glyphotaelien umgeben, die Verhältnisse wieder. Bei hellem Sonnenschein konnte ich in einer Tiefe von $2 \mathrm{~m}$ noch weiße Kieselsteine auf dem Boden der Teiche beobachten.

Nimmt man eine Glyphtotaeliuslarve aus dem Hause, so sinkt sie augenblicklich und schnell hinunter. Die Abfahrt zu dem Boden ca. $(2 \mathrm{~m})$ dauert ohne Röhre ca. 20 Sekunden oder weniger. Das Tier weiB, was es gilt, es krümmt sich, streckt den Vorderkörper aufwärts, keine Potamogetonstengel bieten sich als Unterstützungsflächen dar; - dem Bodenund in den meisten Fällen einem schnellen Tod in einem Fischmagen entgeht es nicht.

Nimmt man nun den Köcher allein, dann schwimmt er, wenn er von Potamogetonblättern gebaut ist, immer an der Oberfläche. Der Köcher macht also die Larve leichter, er kann als eine Korkseele betrachtet werden. Es fragt sich nun, wie viel leichter er das Tier macht; ist er imstande, die Larve zu tragen, und den ursprünglichen Bodenorganismus zu

1) Vereinzelt bis Mitte Juli. 
einem pelagischen Oberflächenorganismus abzuändern? Untersuchen wir näher die Köcher tragenden Larven, so zeigt es sich, daß einige an der Oberfläche schwimmen, andere aber zu Boden sinken. Eine sinkt relativ schnell, eine andere langsam, immer aber ist die Sinkgeschwindigkeit geringer als bei den Tieren ohne Kö̈cher; die zwei Meter werden nie in weniger Zeit als $1^{1} \%$ Minuten zurüekgelegt; immer fällt das Tier auf einer der Breitseiten; sehr oft geht der Fall in einer Schraubenlinie vor sich, dann und wann in einer rechts und links schaukelnden Bewegung. Mehrere stehen in ea. $1 \mathrm{~m}$ Tiefe über 5 Minuten vollkommen ruhig schwebend, Betrachten wir die Oberfläche des Wassers, so finden wir zwischen den Potamogetonblättern frei schwimmende Köcher mit Larrven; immer liegen sie an der Oberfläche und immer mit einer der Breitseiten aufwärts. Ich erinnere mich, daß ich Anfang September sehr zahlreiche schwimmende Köcher sah; im Oktober ist ihre Anzahl nicht so groß, denn jetzt kriechen die meisten Larven an den Blattstielen.

Es fragt sich also nun: Warum sinkt die eine, eine andere nicht? Warum ist das Schwebevermögen bei den Tieren verschieden? Schon früher habe ich bemerkt, daß die Köcher oft Luft enthalten und denke daher, daß verschiedene Luftmenge die Ursache ist. Um dieses näher zu prüfen, nahm ich Tiere, die ca. $1 / 2$ dem unter der Oberfläche sitzen, näherte dieselben bis an die Oberfläche und drückte die Luft mit den Fingern unter dem Wasser aus. Es zeigt sich dann, daß auch diese Tiere, trotzdem sie keine Luft mehr abgeben, dennoch aufwärts steigen können. Dals die Luftmengen in dem Köcher natürlich ein Heruntersinken verhindern, versteht sich von selbst, daß es sich bei den Tieren um ein regelmäBiges Hereinschaffen von Luft in die Röhren handelt, glaube ich nicht. Die Gegenwart von Luft hat viel mehr das Gepräge von etwas zufälligem. Daß die Luftmengen dennoch nicht ganz ohne Bedeutung sind, geht daraus hervor, was ich nicht immer, doch wiederholt beobachtet habe -, daß Tiere, die ich greifen will, Luftblasen abgeben, worauf sie langsam hinuntersinken.

Indem ich nun die Oberfläche näher beobachtete, sehe ich eine Larve, die sich ein neues Deckstück aus einem Potamogetonblatt ausschneidet. Die Pflanzen und der Köcher der Larve wird mit einem Tropfen weißer Farbe markiert und nach einer Stunde wieder beobachtet: dann ist das neue Stück in den Köcher eingefügt. Das neue frisch grüne Stück ragt, wenn der Köcher in der Oberfläche schwimmt, über die Wasseroberfläche empor, wirft man einen Tropfen Wasser darauf, fließt es wie auf den frischen Potamogetonblättern ab. Wird das neue Stück abgenommen, sinkt der Köcher mit der Larve langsam, aber sicher zu Boden. Indem ich nun wieder zahlreiche Köcher studiere, zeigt es sich, daß, so lange die Blätter und besonders das letztangebrachte Blattstück frisch ist, das Tier sich an der Oberfläche halten kann. Wenn das Blatt älter und brüchig wird, wenn 
es mit Wasser durchtränkt ist und seinen Luftinhalt abgegeben hat, vermag das Tier es nicht. Ich bemerkte nun auch, was ich früher übersehen habe, daß die Blattstücke immer die Oberseite aufwärts kehren: dies ist leicht begreiflich, weil das frische Blattstück nur dabei als Adhäsionsapparat verwendet werden kann. Das Tier muB also immer neue Stücke einfügen; wenn die Stücke alt werden, vermag das Tier sich nicht mehr an der Oberfläche zu halten, es muß sich dann damit begnügen auf den Blattstielen und auf den Blättern zu kriechen, und wird es hiervon von Wellen oder Tieren weggeschlagen, muß es hinuntersinken.

Es ist nun ganz klar, daß die Tiere besser an der Oberfläche schwimmen, je größer die Blattstücke sind; die großen Blattstücke sind daher als ein sehr brauchbares Bauprinzip anzusehen. Wer aber diese großen Köcher langsam durch das $W$ asser und immer mit einer den Breitseiten abwärts, schraubenförmig, langsam verschwinden sieht, bekommt unwillkürlich den Eindruck, daß diese eigentümliche regelmäßige Abflachung des Körpers auch eine andere Bedeutung hat. Was über das Rohr herausragt, ist als flügelförmige Fortsätze anzusehen, wodurch das Tier seinen Querschnittswiderstand sehr stark vergrößert. Sie verzögern den Fall, sie ändern die Fallrichtung von einer vertikalen zu einer Schraubenlinie, und haben daher für die Glyphotaeliuslarve dieselbe Bedeutung wie Fallmembranen für die Planktontiere. 1905, da ich zuerst diese großen Köcher an der Oberfläche schwimmen sah, faßte mich augenblicklich der Gedanke: das Bauprinzip der Larven ist Oberflächenvergrößerung und Vergrößerung des Querschnitts, hervorgebracht, um den Fall durch das Wasser zu vermindern. Was die Fallmembranen der Planktonorganismen anbelangt, ist unsere Auffassung dieser Membranen eigentlich nur eine Theorie, die allerdings sehr wahrscheinlich ist; hier war ein Fall, wo ich, der eben damals sehr stark über Planktonorganismen arbeitete, diese Theorie experimentell untersuchen konnte.

In einem $1 / 2 \mathrm{~m}$ hohem Zylinderglas braucht das Tier, um ohne Köcher den Boden zu erreichen, ca. 4 Sekunden oder noch weniger Zeit. Mit unverletztem Köcher brauchen die Tiere, die überhaupt sinken können, eine sehr verschiedene Zeit, wenigstens 10 bis 20 Sekunden. Wenn man nun dem Rohr alle Deckstücke abnimmt, sinkt das Tier immer zu Boden, und immer in einer Linie, die sich viel mehr der vertikalen Linie nähert, als wenn das Rohr mit Deckstücken bekleidet war. Die Zeit ist auch hier sehr verschieden, doch nur wenig länger als wenn das Tier nackt durch das Wasser sinkt.

Daß die großen Flügelfortsätze an den Seiten des Rohres als horizontale Fallmembranen während des Falles also verzögernd wirken, ist, was die Glyphotalienlarven anbelangt, über jedem Zweifel erhaben; und die 
Berechtigung ähnlichen Fallmembranen bei Planktonorganismen dieselbe Bedeutung zuzuteilen ist wieder bekräftigt worden.

Warum verläßt nun das Tier im November--Dezember diesen Bauplan, ,zieht die Fallmembran ein" und verfertigt sich aus Stielstücken ein zylindrisches Rohr? Weil es num ein Bodentier sein soll und daher keinen Gebrauch für Fallmembranen haben kann? Sicherlich nicht. Nur weil die Potamogetonblätter verwesen und weder als Schwimmapparat, noch als Nahrung brauchbar sind. Daher werden die Larven gezwungen, ihren Aufenthalt auf den Blattstielen - die sich länger als die Blätter frisch halten - zu nehmen. Solange diese Blattstiele frisch sind, sind sie so luftgefüllt, daß das Tier auch von ihnen sich einen schwimmenden Köcher konstruieren kann; dagegen ist es augenscheinlich unmöglich, diese schmalen zylindrischen Stücke in der Querrichtung anzubringen und sie nach den Prinzipien eines vergrößerten Querschnitts zu verwenden. Die Änderung der Bauprinzipien ist daher gar nicht als eine Zweckmäßigkeit anzusehen; eine solche kommt nur scheinbar zustande, weil das Tier von den verschiedenen Teilen derselben Pflanze zu verschiedenen Jahreszeiten sowohl seine Baumaterialien als anch seine Nahrung - im Sommer Blätter, im Herbst durch die Verhältnisse gezwungen nur Stiele - nimmt.

Etwas anderes ist es, ob man nicht in der Anordnung der Blattstücke, wobei ein zylindrisches Rohr zu einer flachen Scheibe wird, eine Anpassung an äußere Faktoren suchen darf. Daß dieser Bauplan auf die hier geschilderte Lokalität von wirklicher Bedeutung für das Tier ist, betrachte ich als unzweifelhaft. Damit ist gar nicht gesagt, daß dieser Bauplan von eben diesen Verhältnissen hervorgerufen ist. Auf zahlreichen Lokalitäten Nordseelands findet man Glyphotaelienröhren, die ebenso abgeplattet sind wie die meines Versuchsteiches, und die dennoch, so weit ich gesehen habe, das ganze Jahr hindurch ausgesprochene Bodentiere sind; das Prinzip, den Querschnittswiderstand des Köchers zu vergrößern, ist daher sicherlich nicht als ein "Planktoncharakter", der von den äußeren Faktoren der pelagischen Region abhängig ist, anzusehen; er hat eine andere uns unbekannte Ursache. Dort aber, wo die äußeren Verhältnisse den Larven erlauben an der Oberfläche zu leben, wird ein solcher Bauplan als Mittel, sich nahe an derselben zu halten mit Vorteil benutzt. DaB es die Konstruktion des Köchers ist, die den Glyphotaelien erlaubt, sich eine neue Heimat auf den Potamogetonwiesen zu suchen, finde ich höchst wahrscheinlich. Nie habe ich auf den Teppichen der Schwimmblätter verschiedener Pflanzen, die in etwa 3 bis $4 \mathrm{~m}$ Tiefe wachsen, andere Phryganeen gefunden als Glyphotaelien, Phryganea grandis und Triaenodes bicolor. - Die übrigen Phryganeen, die ihre Köcher von lebenden Pflanzen bauen, besonders die Limnophiliden, finden sich nicht hier; ihre Heimat sind ganz kleine mit Lemna und Algenteppichen bedeckte Teiche. Die monographische Be- 
arbeitung der Biologie der Glyphotaelien ist hiermit abgeschlossen; über Phryganea grandis und Triaenodes bicolor sind die Untersuchungen auch beinahe abgeschlossen, doch sind noch einige Frühjahrsbeobachtungen nötig ehe sie publiziert werden können.

\section{Nachtrag.}

Ich habe im Winter 1910 an verschiedenen Orten in Nordseeland die Glyphotaelien beobachtet und kann nun mit Sicherheit folgendes hervorheben.

Wo die Köcher im Sommer aus abgewelkten Erlenblättern und anderen Bodenmaterial verfertigt sind, behalten die Köcher den ganzen Winter hindurch dieselbe wohlbekannte oben beschriebene breite Form. Am 17. Febr. 1910 wurden die Ufer des Funkenteiches untersucht; der Wasserstand war dann viel höher als im Herbst; über den überschwemmten Ufern, die im Herbst mit abgewelkten Buchen- und Erlenblättern bedeckt waren, lag nun dickes Eis; nur arn nach Süden gelegenen Ufer war das Eis verschwunden. Dort fand ich zahlreiche herumkriechende Glyphotaelien mit ganz gleichartigen Köchern wie im Herbst. Die großen Löcher in den Buchen- und Erlenblättern zeigten, daß die Tiere den ganzen Winter hindurch an ihren Köchern weiter gebaut haben. Hier in Nordseeland wie vielleicht an den meisten Lokalitäten sind die Glyphotaelien das ganze Jahr hindurch hauptsächlich Bodentiere, die auf den Fontinalisteppichen herumkriechen. Wo aber -- wie im Versuchsteich II - eine breite Zone von Schwimmblättern von Potamogeton natans sich befindet, verlassen im Sommerhalbjahr die Tiere den Boden und leben als Oberflächentiere auf den Potamogetonteppichen. Daß die ursprüngliche Form des Köchers unter diesen neuen Verhältnissen den Larven vorteilhaft ist, ist unzweifelhaft. Wenn sie im Spätherbst die Form der Köcher ändern, geschieht dies nur, weil es infolge des Mangels an Material (Potamogetonblätter) zur dringenden Notwendigkeit wird.

\section{Literaturverzeichnis.}

1778. De Geer, K., Abhandlungen zur Geschichte der Insekten. Übersetzt von Götze. II. S. 368 .

1907. Petersen, E., Bidrag til Fortegnelse over Danmarks Vaarfluer. Entomolog. Meddelelser. Köbenhavn II. R. Vol. III, 2., p. 145.

1902. Siltala, A., Ưber die Metamorphose einiger Phryganeiden und Limnophiliden. Acta Soc. pro Fauna et Flora Fennica, Vol. XXI, S. 1.

1906 a. Siltala, A., Beobachtungen über die Okologie der Trichopterenpuppe. Allgem. Zeitschr. f. wiss. Insektenbiol., Bd. II, S. 88.

1906 b. Siltala, A., Uber den Laich der Trichopteren. Acta Soc. pro Fauna et Flora Fennica, Vol. XXVIII, p. 1.

1907 a. Siltala, A., Trichopterologische Untersuchungen. Nr. 2. Zoolog. Jahrb. Supplement., Bd. IX, S. 308. 
r907b. Siltala, A., Uber die Nahrung dor Trichopteren. Acta Soc. pro fauna et flora Fennica. Vol. XXIX, p. 1.

1899. Struck, R., Neue und alte Trichopteren-Larvengehãuse. Illustr. Zeitschr. f. Entomol., Bd. IV, Nr. 8.

1905. Thienemann, A., Biologie der Trichopterenpuppe. Zoolog. Jahrb., Bd. XXII. Abt. f. Syst., S. 1.

\section{Tafelerklärung (Tafel I).}

Alle Figuren sind mit Abbes Zeichenprisma gezeichnet. Wo nichts anderes angegeben ist, sind die Figuren beinahe in natürlicher Größe gezeichnet.

Fig. 1. Laichmasse am Irisblatt.

"2. Eben ausgebrochene Larve mit longitudinaler Rohrbedeckung und einem einzelnen quergelegten Deckstūck. Vergrößerung ca. 10.

$\leadsto 3$.

„4. Köcher aus quergelegten Carexblättern.

" 5.

$" 6 . \quad$ teils aus Carex, teils aus Potamogeton natans.

"7-13. " aus P. natansblättern Fig. 10 von der "Unterseite" gesehen. Fig. 11

nachdem die Deckstücke der einen Seite abgenommen sind.

" 14-18, $n$ teils aus P. natansblättern teiIs aus P. natansblattstielen gebaut. In

"19-20. $n$ hauptsächlich von P. natansblattstielen, zugleich (in Fig. 19) mit einem

Rest von einem submersen Blatt von Scirpus.

"21-22. " aus abgewelkten Pflanzenteilen gemacht.

"23. $"$ in Vorbereitung zu Puppengehäuse.

"24. Faltiges Puppengehäuse.

"25. Querdurchschnitt durch einen Köcher von P. natansblättern.

" 26. Eben ausgekrochene Larve. Vergrößerung ca. 20.

" 27. Die letzten Segmente mit den Nachschiebern derselben. Vergrößerung ca. 60.

"28. Ausgewachsene Larve: Vergleiche die Differenz in der Länge der Beine und Nachschieber bei Fig. 26 und 28.

29. Freischwimmende Puppe.

"30. Vordere Schließmembran des Puppengehäuses. Vergrößerung ca. 10.

"31. Hintere

"32. Imago.

Vergrößerung ca. 10. 